\title{
RF-Powered Systems Using Steep-Slope Devices
}

\author{
Xueqing Li, Unsuk Dennis Heo, Kaisheng Ma, and \\ Vijaykrishnan Narayanan \\ Department of Computer Science and Engineering \\ Penn State University, State College, PA, 16802, US \\ \{xzl3, uih5002,kxm505\}@psu.edu, vijay@cse.psu.edu
}

\author{
Huichu Liu, Suman Datta \\ Department of Electrical Engineering \\ Penn State University, State College, PA, 16802, US \\ hx1249@psu.edu, sdatta@engr.psu.edu
}

\begin{abstract}
Steep-slope tunnel devices promise new opportunities in ultra-low-power computing. This paper focuses on how steep-slope devices can enhance efficiencies of harvesting ambient RF energy and improve power efficiency of analog and digital computational blocks.
\end{abstract}

\section{INTRODUCTION}

Ambient radio-frequency (RF) signals have already been harvested as a power source in applications like passive radio frequency identification (RFID) tags [1]-[10]. The ambient RF signal resonates with the antenna and the receiver circuits in the tag, and powers the signal-processing and wireless data transmission between the battery-free tag and the reader. By replacing batteries with RF-power harvesters, RF-powered systems can reduce cost and weight associated with batteries, and also eliminate the inconvenience of charging or replacing it. For implantable biosensors, the removal of batteries from the system also reduces chemical and biological infection risk.

Due to limited RF power that could be harvested from the ambient environment and the challenges in achieving high power-conversion efficiency (PCE) of energy scavenging from these weak sources, existing RF-powered systems have limited operation range and computational capability. For example, with a wireless several-watt RF signal transmitter, existing RFID tags typically have an operation range of meters and support only primitive sensing, identification, or transmission [1]-[10]. Therefore, exploration of RF-powered systems with more power-efficient energy harvesters, signal processing modules, and wireless transmitters is of great significance.

The advent of new transistor technology promises new opportunities for designing RF-powered systems. Steep-slope tunnel field-effect transistors (TFETs) have become a promising candidate to replace CMOS FETs with higher power-efficiency for low-voltage digital applications due to the tunneling characteristics at a low power supply [11][12][13]. Analog and RF TFET designs have been explored, showing the advantage resulting from features such as lower sub-threshold swing, uni-directional tunneling, and low-voltage operation [12][14]. When steep-slope TFETs are used in the design of RF-powered systems, this paper shows that both the PCE of power harvesters, and the power-efficiency of signal processing blocks can be improved significantly. Such improvements can enable new wireless applications such as insect behavior monitoring, implantable medical devices and swarms of computational sensors.

This work was supported in part by the NSF ASSIST Nanosystems ERC EEC-1160483, and also by LEAST, one of the six SRC STARnet Centers, sponsored by MARCO and DARPA. The NSF award 1317560 supported

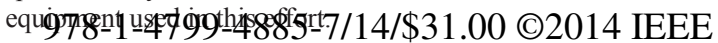

The rest of this paper focuses on the following three aspects: Section II introduces ambient RF power sources. It also provides a review of emerging RF-powered embedded systems and applications with typical power-harvesting and signalprocessing blocks; Section III shows the benefits of employing steep-slope TFET in the RF-powered system design, through the discussions of the device characteristics, and the demonstrations of some key blocks based on steep-slope TFET, to achieve a higher power efficiency in energy harvesting and signal processing. Next, we discuss other challenges in future RF-powered embedded system design using steep-slope TFETs.

\section{AMBIENT RF POWER AND RF-POWERED SYSTEMS}

Typical ambient RF power sources mainly include cellular signals, WiFi, TV broadcast signals, and RFID signals subject to the regulations of the government (e.g. FCC) with varying frequency and power density. Assuming ideal impedance match between the antenna and the power harvesting circuit, Fig. 1 summarizes the sensed RF power density in the urban area, in comparison with typical circuit power consumptions.

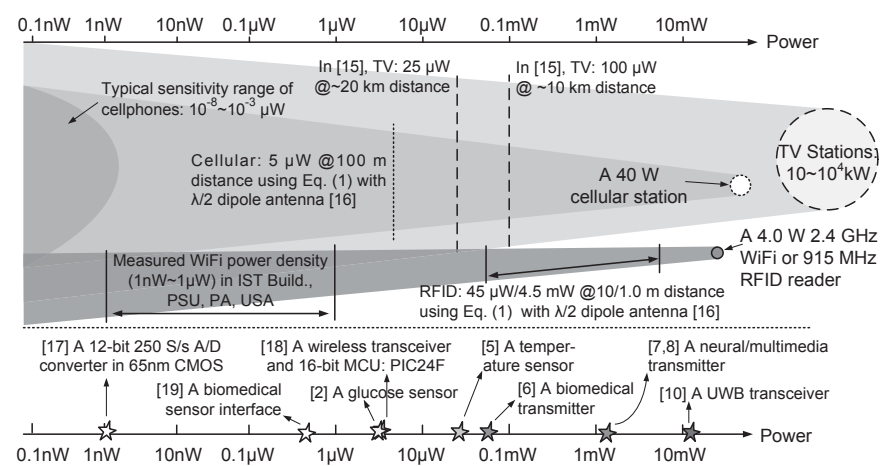

Fig. 1. Ambient RF power compared with typical circuit power consumptions.

\section{A. Power Obtained By the Receiver Antenna}

In order to calculate the harvested power by the receiver antenna $P_{r}$ from RF signals, the Friis free-space transmission equation gives $P_{r}$ at a distance $d$, with the transmitted power $P_{t}$ :

$$
P_{r}=P_{t} \cdot G_{t} \cdot G_{r} \cdot \eta_{\text {harvester }} \cdot(\lambda / 4 \pi d)^{2},
$$

where $G_{t}$ and $G_{r}$ are the antenna gains with respect to an isotropic radiator of the transmitting and receiving antennas respectively, $\lambda$ is the wavelength, and $\eta_{\text {harvester }}$ is the PCE of the power harvester consisting of impedance matching network and RF-to-DC rectifier [16]. It is assumed that the impedance matching is ideal to make sure the power obtained by the 
TABLE I. RECENTLY REPORTED RF-POWERED SYSTEMS

\begin{tabular}{|c|c|c|c|c|c|c|c|c|}
\hline & {$[2]$} & [3] & {$[5]$} & [6] & [7] & [8] & [9] & {$[10]$} \\
\hline Year of publication & 2012 & 2012 & 2010 & 2011 & 2012 & 2014 & 2013 & 2011 \\
\hline System function & $\begin{array}{l}\text { Glucose } \\
\text { sensor }\end{array}$ & $\begin{array}{l}\text { Battery } \\
\text { charging }\end{array}$ & $\begin{array}{c}\text { Temperature } \\
\text { sensor }\end{array}$ & $\begin{array}{l}\text { Biomedical } \\
\text { transmitter }\end{array}$ & $\begin{array}{l}\text { Neural/EM } \\
\text { G telemetry }\end{array}$ & $\begin{array}{c}\text { Wireless } \\
\text { transceiver }\end{array}$ & $\begin{array}{l}\text { Audio/Image } \\
\text { transmission }\end{array}$ & $\begin{array}{l}\text { Wireless } \\
\text { transceiver }\end{array}$ \\
\hline $\begin{array}{l}\text { RF signal } \\
\text { sensitivity }\end{array}$ & $\begin{array}{c}\text { 15cm@ } \\
10 \text { WIRP }\end{array}$ & / & $-12 \mathrm{dBm}$ & $\begin{array}{c}-6 \mathrm{dBm} @ \\
918 \mathrm{MHz}\end{array}$ & $\begin{array}{l}1.5 \mathrm{~m} @ \\
\text { 4W EIRP }\end{array}$ & $-17.1 \mathrm{dBm}$ & $\begin{array}{c}4 \mathrm{~m} @ 4 \mathrm{~W} \\
\text { EIRP }\end{array}$ & $\begin{array}{c}14 \mathrm{dBm} @ \\
900 \mathrm{MHz} \\
\end{array}$ \\
\hline Carrier frequency & $1.8 \mathrm{GHz}$ & $950 \mathrm{MHz}$ & $900 \mathrm{MHz}$ & $\begin{array}{l}918 \mathrm{MHz}, \\
306 \mathrm{MHz}\end{array}$ & $915 \mathrm{MHz}$ & $\begin{array}{l}915 \mathrm{MHz} \\
2.45 \mathrm{GHz}\end{array}$ & $915 \mathrm{MHz}$ & $7.9 \mathrm{GHz}$ \\
\hline Modulation scheme & FM-LSK & 1 & EPC & OOK & BPSK & FSK/OOK & BPSK & UWB \\
\hline Process & $\begin{array}{c}0.13 \mu \mathrm{m} \\
\text { CMOS }\end{array}$ & $\begin{array}{c}0.18 \mu \mathrm{m} \\
\mathrm{CMOS}\end{array}$ & $\begin{array}{c}0.13 \mu \mathrm{m} \\
\mathrm{CMOS}\end{array}$ & $\begin{array}{c}0.13 \mu \mathrm{m} \\
\text { CMOS }\end{array}$ & $\begin{array}{c}0.35 \mu \mathrm{m} \\
\text { CMOS }\end{array}$ & $\begin{array}{l}90 \mathrm{~nm} \\
\text { CMOS }\end{array}$ & PCB & $\begin{array}{l}0.13 \mu \mathrm{m} \\
\mathrm{CMOS}\end{array}$ \\
\hline Power consumption & $3 \mu \mathrm{W}$ & 1 & $16-33 \mu \mathrm{W}$ & $50.6 \mu \mathrm{W}$ & $1.23 \mathrm{~mW}$ & $0.85 \mathrm{~mW}$ & $1.23 \mathrm{~mW}$ & $10.9 \mathrm{~mW}$ \\
\hline $\begin{array}{c}\text { Energy harvesting } \\
\text { efficiency }\end{array}$ & $20 \%$ (peak) & $\begin{array}{c}40 \% @-11 \\
\mathrm{dBm} \text { input }\end{array}$ & $36.6 \%$ & $20-30 \%$ & $20.6 \%$ & / & $20.6 \%$ & / \\
\hline Data rate & 1 & 1 & 1 & $4 \mathrm{Mbps}$ & $5 \mathrm{Mbps}$ & $5 \mathrm{Mbps}$ & $5 \mathrm{Mbps}$ & $112 \mathrm{Mbps}$ \\
\hline
\end{tabular}

antenna is absorbed by the subsequent rectifier, which converts $\mathrm{RF}$ input signal to DC output voltage. Note that the received power $P_{r}$ decreases with the square of the frequency and the distance $d$. Also, the ideal size of a certain type of antenna is proportional to $\lambda$. If it cannot be satisfied by some sizerestricted applications, the harvested power would be less.

\section{B. RF-Powered Systems And Applications}

In recent years, many RF-powered applications have emerged, including battery chargers [3], wideband transceivers [10], and various sensing systems with temperature sensors [5], pressure sensors [1], neural sensors [7], and glucose sensors [2]. Table I lists some recently reported RF-powered systems. These systems harvest power from RF signals in the ultra-high frequency (UHF) band, and the consumed power ranges from a few micro watts for low-power biomedical sensors to higher than ten milliwatts for a high-speed UWB transceiver with a data rate up to $112 \mathrm{Mbps}$. The operation ranges of the systems in Table I are restricted by the system power consumption and low power-harvesting efficiency. From (1), it can be easily derived that if the power-harvesting efficiency is doubled and the power consumed is halved, the operation range doubles.

Fig. 2 shows the structure of typical RF-powered systems. It mainly consists of the power harvesting and management block, analog/RF frontend and digital processing and storage block, and the sensors. The rectifier is critical in the system design because its PCE directly affects the power budget of the entire system. The DC-DC converter in Fig. 2 is an option to boost the output voltage of the rectifier, the low dropout regulator (LDO) is employed to keep the supply voltage stable and less noisy, and the on-chip energy storage (e.g. a capacitor) is necessary to enable transient large current.

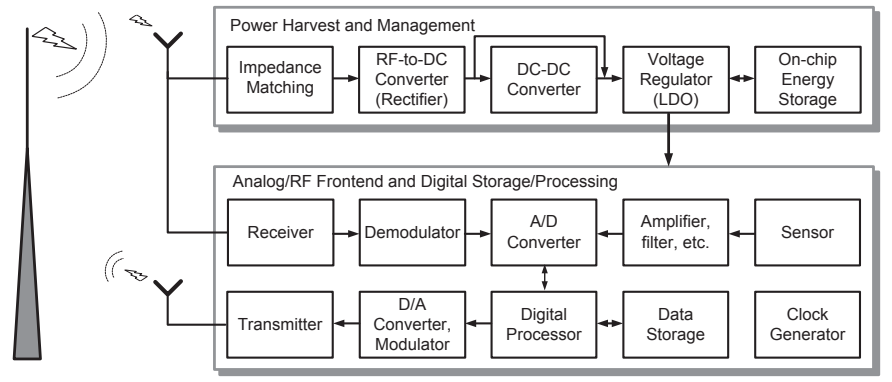

Fig. 2. RF-powered system structure.
The second part of the RF-powered system in Fig. 2 contains some blocks that are necessary only in specific applications. For example, the receiver, demodulator, amplifier, and the data storage depend on the function of the system. Backscattering-based transmitters are widely used to guarantee low-power consumption [5]-[10][17], reaching $<10 \mathrm{pJ} /$ bit [6]. When generating the local clock, a trade-off exists between the large tuning range, high phase noise of ring oscillators and the large area, small tuning range, relatively higher power of LC oscillators. Recent works in [6][8] exploit the RF input signal as a reference frequency to generate a different carrier frequency to transmit the data, which avoids the use of a local crystal oscillator, achieves highly integrated low-cost wireless transceivers, and also eliminates the "self-jamming" problem presented to RFID readers by the backscattering solution.

\section{BENEFITS In RF-PowERED EMBEDDED SYSTEM DESIGN USING STEEP-SLOPE TUNNEL FETS}

Steep-slope TFETs have been proposed to further scale the power supply in ultra-low-power applications [11]. A TFET is essentially reverse-biased, gated $\mathrm{p}-\mathrm{i}-\mathrm{n}$ tunnel diode with asymmetrical source/drain doping, whose on-state drain-source current is enabled by gate-controlled band-to-band tunneling, and off-state current is determined by the reverse-biased diode leakage [11][12][13].
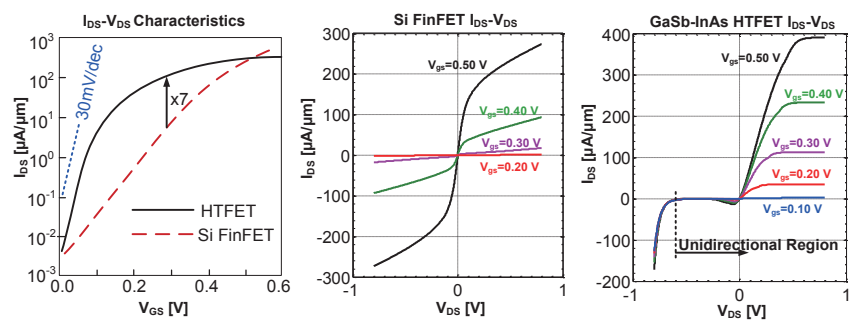

Fig. 3. I-V comparisons for Si FinFET and GaSb-InAs HTFET [12][14][25].

Steep-slope Switching. The tunneling mechanism makes TFET significantly different from CMOS, and the subthreshold swing (SS) of TFET is not limited to $60 \mathrm{mV} /$ decade as in CMOS. With the utilization of low-bandgap materials (e.g. Ge, III-Vs, etc) with a low effective mass $\left(\mathrm{m}^{*}\right)$, tunnel junctions with steep profiles and less defects, and improved gate-control from planar structures towards gate-all-around structures, the SS can be further improved and the on-state current can be 
nearly the same as MOSFETs [12][21]-[23]. It has been reported that III-V heterojunction tunnel field-effect transistor (HTFET) exhibits higher energy efficiency than the start-ofthe-art CMOS technology at a power supply lower than $0.5 \mathrm{~V}$ [24]. As shown in Fig. 3, for low-power applications with offstate leakage of $5 \mathrm{nA} / \mathrm{um}$, the HTFET technology in this paper shows an average $\mathrm{SS}$ of $30 \mathrm{mV} /$ decade over two decades of current, and seven times improvement of on-state current over $20 \mathrm{~nm}$ Si FinFETs at a $0.30 \mathrm{~V}$ power supply [13][14][25]. Due to such characteristics, HTFET has presented a potential for low-voltage applications. Simulation results of the energydelay performance comparison in Fig. 4 confirm such conclusions [25]. For analog applications, steep-slope TFETs also achieve a higher $\mathrm{g}_{\mathrm{m}} / \mathrm{I}_{\mathrm{DS}}$ than MOSFET, representing a larger effective gain per energy step [12][14].
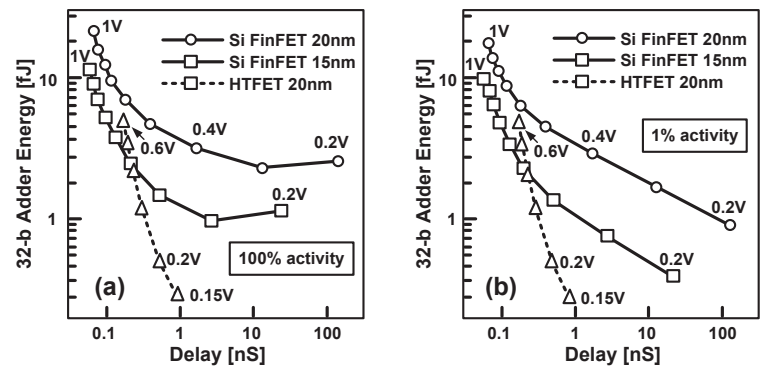

Fig. 4. 32-b Han-Carlson adder energy-delay evaluation for HTFET and Si FinFET at $100 \%$ activity in (a) and $1 \%$ activity in (b) [25].

Uni-directional Tunnel Conduction. Another HTFET feature different from MOSFET is the uni-directional tunnel conduction due to the asymmetrical p-i-n structure [12][13]. With reduced drain doping to restrain the ambipolar transport, when the $\mathrm{p}-\mathrm{i}-\mathrm{n}$ diode is forward-biased within $-0.4 \mathrm{~V}<$ $\mathrm{V}_{\mathrm{DS}}=\mathrm{V}_{\text {neg }}<0 \mathrm{~V}$, the drain-source current is several orders lower than the current when $\mathrm{V}_{\mathrm{DS}}=\left|\mathrm{V}_{\text {neg }}\right|$, as shown in Fig. 3.

The steep-slope I-V curves and the uni-directional tunnel conduction give rise to new features for power harvesting and mixed-signal circuits. The rest of this section will focus on some key blocks of the RF-powered systems with comparisons between $20 \mathrm{~nm}$ GaSb-InAs HTFET and Si FinFET designs.
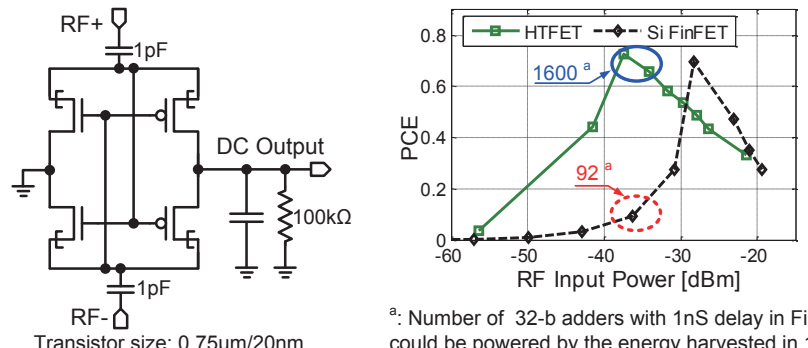

a. Number of $32-\mathrm{b}$ adders with $1 \mathrm{nS}$ delay in Fig. 4 that could be powered by the energy harvested in $1.0 \mu \mathrm{S}$.

Fig. 5. Comparisons between HTFET and Si FinFET one-stage rectifiers.

\section{A. Rectifiers Using HTFET}

Charge-pumps are usually employed to convert the input $\mathrm{RF}$ signal to a DC output voltage. Considering the amplitude of the input RF signal is small when the received power is low, small turn-on voltage is one important factor. In conventional designs, Schottky diode has thus been utilized because of small turn-on voltage [26]. With steep switching, improved $\mathrm{I}_{\mathrm{ON}}$, and uni-directional conduction to reduce the leakage loss, the
HTFET high-sensitivity rectifier in [13] shows much higher PCE than Si FinFET design when the input power is low, as shown in Fig. 5. Furthermore, as the RF-powered adder number comparison in Fig. 5 reveals, with power-efficient digital cells, an HTFET RF-powered system supports much more functions than a Si FinFET RF-powered system.

\section{B. DC-DC Converters Using HTFET}

To boost the power supply voltage, switched-capacitance DC-DC converters make use of charge-pumps under the control of square waves, rather than the sinusoidal wave in rectifiers. As a result, the switching operations in DC-DC converters have lower "on-state" switch resistance to achieve less voltage drop across the switches, and higher "off-state" switch resistance to achieve less current leakage. Therefore, reported PCE of DC-DC converters are generally higher than that of rectifiers. Using a similar circuit topology of rectifier, simulation results in Fig. 6 show that the HTFET DC-DC converter is able to convert an input voltage as low as $0.20 \mathrm{~V}$ with higher than 90\% PCE, while the Si FinFET has a PCE lower than $60 \%$ when the input voltage is lower than $0.35 \mathrm{~V}$.
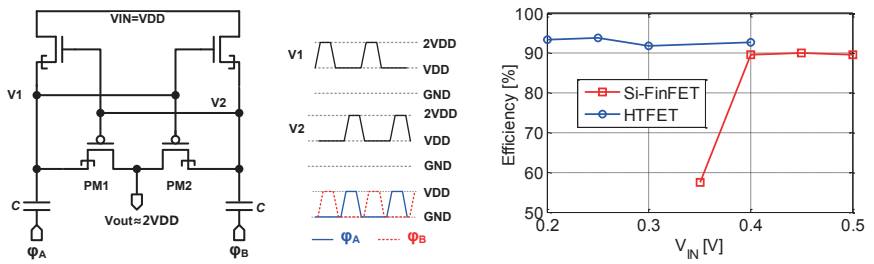

Fig. 6. Ultra-low-voltage, high-efficiency HTFET DC-DC converter.

\section{Low-Power Low-Noise Amplifiers Using HTFET}

Amplifiers are widely used in sensor nodes. For example, human brain neuron action capture requires an array of neuron signal amplifiers. Considering typical neuron spikes have a small amplitude and a frequency range of several $\mathrm{kHz}$, low noise and high gain are key specifications of the amplifier design [19]. Also, considering many neuron amplifiers are integrated on a small chip inside the skull, low-power consumption is also extremely important so as not to excessively heat the brain. In [27], it is presented that high intrinsic gain, driving ability, and output resistance of HTFET can be used to achieve superior mixed-signal performance over Si FinFET design, at an improved electrical noise performance.

\section{Other Blocks}

It has also been observed that the significant power savings in the digital circuits due to low voltage operation are particularly valuable in circuits such as successive approximation register (SAR) A/D converters which consume most of the power in the digital SAR logic and the comparators [17]. Our ongoing efforts show that HTFET SAR A/D converter achieves approximately 3 times power consumption reduction and 6 times size reduction. The simulated effective number of bits (ENOB) of HTFET SAR A/D converter also outperforms its Si FinFET counterpart.

\section{Future RF-Powered System DESIGN CHALLENGES}

The advantage of employing HTFET in low-power RFpowered system has been demonstrated with high power 
efficiency in both energy-harvesting and signal processing. However, RF-powered systems still face the challenge of weak and unstable ambient RF signals, under which the operations of RF-powered systems may be interrupted. Furthermore, for some applications like the glucose sensor on eye contact-lens and the neuron sensors on a flying insert, the size and weight of the energy-harvesting antenna are restricted, which reduces the design space of the energy-harvester to obtain sufficient power. This condition makes the design of a RF-powered system with a wide operation range much more challenging.

Further reduction of the digital and analog/RF power consumption helps to mitigate the impact of insufficient harvested power. Other approaches include the use of hybrid power sources, and intermittent operations to gather enough energy before the next operation starts. Nonvolatile memorybased processors are being explored to support such intermittent computing systems [28]-[30].

\section{CONCLUSIONS}

In this paper, we have reviewed the ambient RF signals, as well as the emerging RF-powered systems and applications. It has been confirmed that the power efficiency of the energy harvesting and signal processing is the key metric for lowpower RF-powered systems. We have demonstrated the benefits of utilizing the TFET characteristics including the steep-slope current driving ability, uni-directional tunneling mechanism, etc., in the RF-powered systems. A series of key blocks, i.e. rectifier, DC-DC converter, low-power amplifier, and A/D converter, are also presented with superior performance of power-harvesting efficiency, mixed-signal performance over Si FinFET designs. The challenges in future RF-powered embedded systems are also discussed, along with proposed approaches to mitigating the impact of insufficient and intermittent harvested power.

\section{REFERENCES}

[1] D. J. Young, "An RF-powered wireless multi-channel implantable biosensing microsystem," in IEEE Engineering Medicine and Biology Society (EMBC), Annual Int. Conf., Sept. 2010, pp. 6413-6416.

[2] Y. T. Liao, "A $3 \mu \mathrm{W}$ CMOS glucose sensor for wireless contact-lens tear glucose monitoring," IEEE J. Solid-State Circuits, vol. 47, no. 1, pp. 335-344, Jan. 2012.

[3] M. Arrawatia, V. Diddi, H. Kochar, M. S. Baghini, and G. Kumar, "An integrated CMOS RF energy harvester with differential microstrip antenna and on-chip charger," in VLSI Design (VLSID), 25th Int. Conference on, Jan. 2012, pp. 209-214.

[4] N. Cho et al, "A $8-\mu \mathrm{W}, 0.3-\mathrm{mm}^{2}$ RF-powered transponder with temperature sensor for wireless environmental monitoring," in IEEE Int. Circuits Syst Symp., May 2005, pp. 4763-4766.

[5] D. Yeager, Z. Fan, A. Zarrasvand, N. T. George, T. Daniel, and B. P. Otis, "A $9 \mu \mathrm{A}$, addressable Gen2 sensor tag for biosignal qcquisition," IEEE J. Solid-State Circuits, vol. 45, no. 10, pp. 2198-2209.

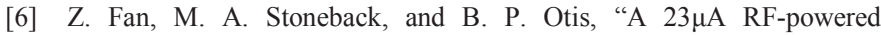
transmitter for biomedical applications," in IEEE Int. RFIC Symp., Jun. 2011, pp. 1-4.

[7] S. J. Thomas et al, "A battery-free multichannel digital Neural/EMG telemetry system for flying insects," IEEE Trans. Biomedical Circuits and Syst., vol. 6, no. 5, pp. 424-436, Oct. 2012.

[8] G. Papotto et al., "A 90-nm CMOS 5-Mbps crystal-less RF-powered transceiver for wireless sensor network nodes," IEEE J. Solid-State Circuits, vol. 49, no. 2, pp. 335-346, Feb. 2014.
[9] S. J. Thomas et al, "Rich-media tags: battery-free wireless multichannel digital audio and image transmission with UHF RFID techniques," in IEEE Int. RFIC Symp., April 2013, pp. 231-236.

[10] M. Pelissier et al, "A $112 \mathrm{Mb} / \mathrm{s}$ full duplex remotely-powered impulseUWB RFID transceiver for wireless NV-memory applications," in IEEE VLSI Circuits Symp., Jun. 2010, pp. 25-26.

[11] Seabaugh, A. C. et al, "Low-voltage tunnel transistors for beyond CMOS Logic," in IEEE Proc, vol. 98, iss.12, pp. 2095-2110, 2010.

[12] Huichu Liu et al, "Steep Switching Tunnel FET: A promise to extend the energy efficient roadmap for post-CMOS digital and analog/RF applications," In IEEE ISLPED, Sept. 2013, pp. 145-150.

[13] Huichu Liu et al, "Exploration of vertical mosfet and tunnel FET Device architecture for sub 10nm node applications," In 70th Annual Device Research Conference (DRC), June 2012, pp. 233-234.

[14] Huichu Liu et al, "Tunnel FET-based ultra-low power, high-sensitivity UHF RFID Rectifier," In IEEE International Symp. on Low Power Electronics and Design, Sept. 2013, pp. 157-162.

[15] A. N. Parks et al, "A wireless sensing platform utilizing ambient RF energy," in IEEE Topical Conference on Wireless Sensors and Sensor Networks (WiSNet), Jan. 2013, pp. 127-129.

[16] H. T. Friis, "A note on a simple transmission formula," Proc. IRE, vol. 34, no. 5, pp. 254-256, May 1946.

[17] Pieter Harpe, Eugenio Cantatore, and Arthur van Roermund, “A 10b/12b $40 \mathrm{kS} / \mathrm{s}$ SAR ADC with data-driven noise reduction achieving up to 10.1b ENOB at $2.2 \mathrm{fJ} /$ conversion-step," IEEE J. Solid-State Circuits, vol. 48, no. 12, pp. 3011-3018, Dec. 2013.

[18] R. J. Vyas et al, "E-WEHP: a batteryless embedded sensor-platform wirelessly powered from ambient digital-TV signals," IEEE Trans. Microw. Theory Tech., vol. 61, no. 6, pp. 2491-2505, June 2013.

[19] Xiaodan Zou, Xiaoyuan Xu, Libin Yao, and Yong Lian, "A 1-V 450-nW fully integrated programmable biomedical sensor interface chip," IEEE J. Solid-State Circuits, vol. 44, no. 4, pp. 1067-1077, April 2009.

[20] S. J. Thomas et al, "Quadrature amplitude modulated backscatter in passive and semi-passive UHF RFID systems," IEEE Trans. Microw. Theory Tech., vol. 60, no. 4, pp. 1175-1182, Apr. 2012.

[21] Zhou, G. et al, "Novel gate-recessed vertical InAs/GaSb TFETs with record high $\mathrm{I}_{\mathrm{ON}}$ of $180 \mu \mathrm{A} / \mu \mathrm{m}$ at $\mathrm{VDS}=0.5 \mathrm{~V}$," in IEEE IEDM Tech. Dig., Dec. 2012, pp.32.6.1-32.6.4.

[22] Zhou, G. et al, "InGaAs/InP tunnel FETs with a subthreshold swing of $93 \mathrm{mV} / \mathrm{dec}$ and $\mathrm{I}_{\mathrm{ON}} / \mathrm{I}_{\mathrm{OFF}}$ ratio near $10^{6}$," IEEE. Elec. Dev. Lett., vol. 33, no. 6 , pp. $782-784,2012$

[23] Mohata, D. et al, "Demonstration of improved heteroepitaxy, scaled gate stack and reduced interface states enabling heterojunction tunnel FETs with high drive current and high on-off ratio," in Symp. VLSI Tech. Dig., June 2012, pp. 53.

[24] V. Saripalli et al, "Variation-tolerant ultra low-power heterojunction tunnel FET SRAM design," in IEEE/ACM Int. Nanoscale Architectures (NANOARCH), Symp., Jun. 2011, pp. 45-5.

[25] S. Datta et al, "Tunnel transistors for energy efficient computing," In IEEE Int. Reliability Physics Symp. (IRPS), 2013, pp. 6A.3.1- 6A.3.7.

[26] K. Kotani, A. Sasaki, and T. Ito, "High-efficiency differential-drive CMOS rectifier for UHF RFIDs," IEEE J. Solid-State Circuits, vol. 44, no. 11, pp. 3011-3018, Nov. 2009

[27] R. Pandey, B. Rajamohanan, L. Huichu, V. Narayanan, and S. Datta, "Electrical noise in heterojunction interband tunnel FETs," IEEE Trans. Electron Devices, vol. 61, no. 2, pp. 552-560, Feb. 2014.

[28] Meng-Fan Chang et al, "Embedded 1Mb ReRAM in 28nm CMOS with 0.27 -to-1V read using swing-sample-and-couple sense amplifier and self-boost-write-termination scheme," in IEEE ISSCC Dig. Tech. Papers, Feb. 2014, pp. 332-333.

[29] Y. Wang, Yongpan Liu, Shuangchen Li, Daming Zhang et al, "A $3 \mu \mathrm{s}$ wake-up time nonvolatile processor based on ferroelectric flip-flops," in Proc. ESSCIRC, Sept. 2012, pp. 149-152.

[30] S. Khanna et al, "An FRAM-based nonvolatile logic MCU SOC exhibiting $100 \%$ digital state retention at $0 \mathrm{v}$ achieving zero leakage with 400-ns wakeup time for ULP applications", IEEE J. Solid-State Circuits, vol. 49, no. 1, pp. 95-106, Jan. 2014. 